\title{
Rapid control of severe ectopic Cushing's syndrome by oral osilodrostat monotherapy
}

\author{
Laura Bessiène ${ }^{1,7}$, Fidéline Bonnet ${ }^{2,6,7}$, Florence Tenenbaum ${ }^{3}$, Mathieu Jozwiak ${ }^{4,7}$, Anthony Corchia ${ }^{5}$, \\ Jérôme Bertherat ${ }^{1,6,7}$ and Lionel Groussin ${ }^{1,6,7}$
}

${ }^{1}$ Department of Endocrinology, ${ }^{2}$ Department of Hormonology, ${ }^{3}$ Department of Nuclear Medicine, ${ }^{4}$ Medical ICU, APHP, Cochin Hospital, Paris, France, ${ }^{5}$ Department of Nephrology, Polyclinic of Saint Côme, Compiegne, France, ${ }^{6}$ INSERM Unité 1016, CNRS UMR 8104, Institut Cochin, Paris, France, and ${ }^{7}$ Université de Paris, Paris, France

We read with great interest the recent letter by Haissaguerre et al. who used osilodrostat, a new 11ß-hydroxylase inhibitor, in three patients with Cushing's syndrome due to non-pituitary tumors (1). The reported cases had intense hypercortisolism with initial mean cortisol levels around $1400 \mathrm{nmol} / \mathrm{L}$. No cases required to transfer to an intensive care unit (ICU). The substitution of previous inhibitors with osilodrostat, in combination with ketoconazole or mitotane in two patients, made it possible to control hypercortisolism. Severe Cushing's syndrome is a rare situation that can lead to death (2). The leading causes of mortality are dysmetabolic disorders such as profound hypokalemia uncontrolled diabetes, thromboembolic events, hypertension, cardiovascular diseases, severe infections, and acute psychiatric disorders (3). This critical situation requires emergency treatment and close monitoring. Several therapeutic strategies based on adrenal steroidogenesis inhibitors, such as metyrapone, ketoconazole, etomidate or mitotane can be used $(4,5,6$, 7). Bilateral adrenalectomy may be necessary as a rescue treatment in patients unresponsive to medical therapy (8). Osilodrostat was approved after clinical trials conducted on adults with Cushing's disease $(9,10)$. Its potential as a therapeutic against other causes of Cushing's syndrome remains to be evaluated. To justify its potential interest in severe Cushing's syndrome, we describe the cortisol response to osilodrostat as a first-line monotherapy on a 49-year-old woman admitted to the ICU (Fig. 1). Having read the letter by Haissaguerre, we decided to start osilodrostat with a high dose of $20 \mathrm{mg} /$ day given the severe condition of our patient. To date, the literature on the efficacy of osilodrostat in Cushing's syndrome due to non-pituitary tumors contains the letter by Haissaguerre (1) and a phase 2 study from Japan (11). A total of five ectopic Cushing's syndrome have been reported. None of them required intensive care, unlike our patient with a life-threatening presentation. This is probably the reason why in previous publications osilodrostat titration was performed every 2-5 days (1) or week (11). Normalization of cortisol levels was obtained after 15 and 44 days (1) or at least 4 weeks (11) for the five reported patients with ectopic Cushing's syndrome. Our patient required a more rapidly effective approach, which is why we chose to start with a high dose (20 mg/day) move quickly in 5 days to a block and replace strategy with the maximum recommended dose (60 $\mathrm{mg} /$ day). High dose osilodrostat as a first-line monotherapy normalized the cortisol levels of the patient in only 6 days, allowing a rapid discharge from ICU. ACTH levels remained elevated during osilodrostat treatment, demonstrating that the resolution of hypercortisolism was due to adrenal steroidogenesis inhibition.

A lower dose may have been sufficient, but we gave priority to a rapid decrease in cortisol levels, with the subsequent possibility to reduce osilodrostat as has been done with this patient. Among the rapidly effective options in ICU for severe Cushing's syndrome, etomidate may be used but an i.v. infusion is needed (6). Osilodrostat could be the first option to consider in severe Cushing's syndrome with the advantage of being given orally twice a day. Another option in severe Cushing's syndrome is a combined therapy with several anticortisolic drugs (12). Our observation suggests that this combined strategy could be used as a second-line (c) 2021 European Society of Endocrinology Printed in Great Britain
Published by Bioscientifica Ltd. 


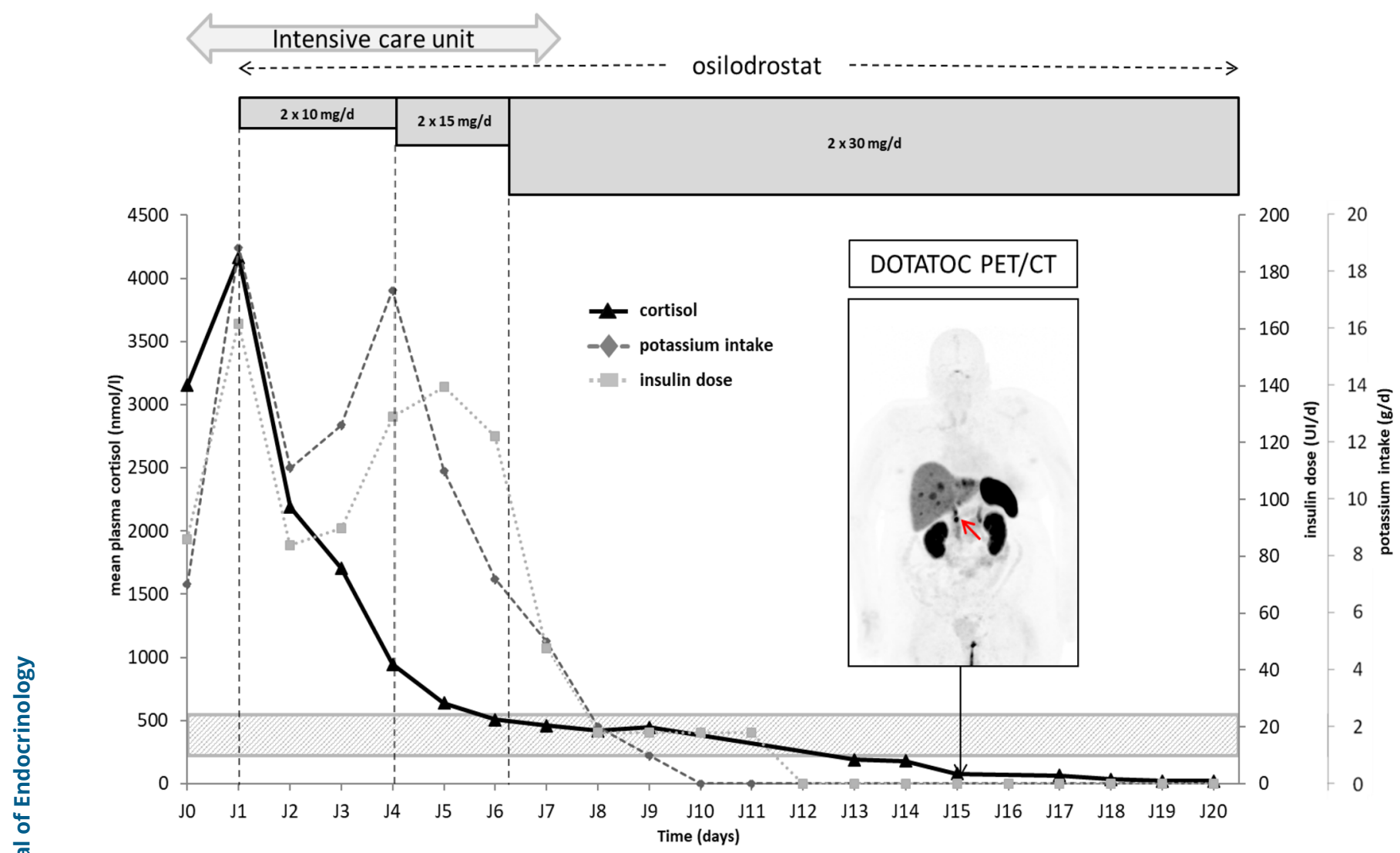

\section{Figure 1}

Evolution of mean plasma cortisol concentrations for a severe ectopic Cushing's syndrome treated with a high dose of osilodrostat. Cortisol values are the mean of 6-day time measurements. The shaded area corresponded to cortisol reference ranges (200-550 nmol/L). The patient was hospitalized in ICU for 6 days due to profound hypokalemia and uncontrolled diabetes mellitus. The gray background steps represent the titration regimen of osilodrostat in $\mathrm{mg} / \mathrm{day}$. Rapid correction of hypercortisolism was followed by a complete blockage of cortisol production obtained with the maximum recommended dose in a 'block and replace' strategy. The initial high i.v. potassium intake and insulin dose declined in parallel with a slight delay. DOTATOC- $\left({ }^{68} \mathrm{Ga}\right)$ PET/CT performed on the 15th day revealed a pancreatic tumor (arrow) with liver metastases from a mixed neuroendocrine non-neuroendocrine tumor (MiNEN).

option in case of osilodrostat ineffectiveness after early cortisol monitoring.

In conclusion, high-dose osilodrostat was rapidly effective, easy to use and well tolerated, as a first-line monotherapy in an extremely severe Cushing's syndrome in ICU. As stated by Haissaguerre et al. complementary observations in these complex therapeutic situations are needed to determine optimal starting doses of osilodrostat and the optimal drug dosing schedule.

\section{Declaration of interest}

The authors declare that there is no conflict of interest that could be perceived as prejudicing the impartiality of this letter.

\section{Funding}

This work did not receive any specific grant from any funding agency in the public, commercial or not-for-profit sector or industry.

\section{References}

1 Haissaguerre M, Puerto M, Nunes ML \& Tabarin A. Efficacy and tolerance of osilodrostat in patients with severe Cushing's syndrome due to non-pituitary cancers. European Journal of Endocrinology 2020 183 L7-L9. (https://doi.org/10.1530/EJE-20-0557)

2 Yaneva M, Kalinov K \& Zacharieva S. Mortality in Cushings syndrome: data from 386 patients from a single tertiary referral center. European Journal of Endocrinology 2013169 621-627. (https:// doi.org/10.1530/EJE-13-0320)

3 Pivonello R, Isidori AM, De Martino MC, Newell-Price J, Biller BM $\&$ Colao A. Complications of Cushings syndrome: state of the art. 
Lancet: Diabetes and Endocrinology 20164 611-629. (https://doi. org/10.1016/S2213-8587(16)00086-3)

4 Young J, Haissaguerre M, Viera-Pinto O, Chabre O, Baudin E \& Tabarin A. Management of endocrine disease: Cushings syndrome due to ectopic ACTH secretion: an expert operational opinion. European Journal of Endocrinology 2020182 R29-R58. (https://doi. org/10.1530/EJE-19-0877)

5 Daniel E \& Newell-Price JD. Therapy of endocrine disease: steroidogenesis enzyme inhibitors in Cushings syndrome. European Journal of Endocrinology 2015172 R263-R280. (https://doi. org/10.1530/EJE-14-1014)

6 Constantinescu SM, Driessens N, Lefebvre A, Furnica RM, Corvilain B $\&$ Maiter D. Etomidate infusion at low doses is an effective and safe treatment for severe Cushings syndrome outside intensive care. European Journal of Endocrinology 2020183 161-167. (https://doi. org/10.1530/EJE-20-0380)

7 Donadille B, Groussin L, Waintrop C, Abbas H, Tenenbaum F, Dugue MA, Coste J, Bertagna X \& Bertherat J. Management of Cushings syndrome due to ectopic adrenocorticotropin secretion with 1,ortho-1, para'-dichloro-diphenyl-dichloro-ethane: findings in 23 patients from a single center. Journal of Clinical Endocrinology and Metabolism 201095 537-544. (https://doi.org/10.1210/jc.2009-1317)

8 Zeiger MA, Fraker DL, Pass HI, Nieman LK, Cutler Jr GB, Chrousos GP $\&$ Norton JA. Effective reversibility of the signs and symptoms of hypercortisolism by bilateral adrenalectomy. Surgery 1993114 $1138-1143$
9 Pivonello R, Fleseriu M, Newell-Price J, Bertagna X, Findling J, Shimatsu A, Gu F, Auchus R, Leelawattana R, Lee EJ et al. Efficacy and safety of osilodrostat in patients with Cushings disease (LINC 3): a multicentre phase III study with a double-blind, randomised withdrawal phase. Lancet: Diabetes and Endocrinology 20208 748-761. (https://doi.org/10.1016/S2213-8587(20)30240-0)

10 Bertagna X, Pivonello R, Fleseriu M, Zhang Y, Robinson P, Taylor A, Watson CE, Maldonado M, Hamrahian AH, Boscaro M et al. LCI699, a potent 11beta-hydroxylase inhibitor, normalizes urinary cortisol in patients with Cushings disease: results from a multicenter, proof-of-concept study. Journal of Clinical Endocrinology and Metabolism 201499 1375-1383. (https://doi. org/10.1210/jc.2013-2117)

11 Tanaka T, Satoh F, Ujihara M, Midorikawa S, Kaneko T, Takeda T, Suzuki A, Sato M \& Shimatsu A. A multicenter, phase 2 study to evaluate the efficacy and safety of osilodrostat, a new 11betahydroxylase inhibitor, in Japanese patients with endogenous Cushings syndrome other than Cushings disease. Endocrine Journal 202067 841-852. (https://doi.org/10.1507/endocrj.EJ19-0617)

12 Kamenicky P, Droumaguet C, Salenave S, Blanchard A, Jublanc C, Gautier JF, Brailly-Tabard S, Leboulleux S, Schlumberger M, Baudin E et al. Mitotane, metyrapone, and ketoconazole combination therapy as an alternative to rescue adrenalectomy for severe ACTH-dependent Cushings syndrome. Journal of Clinical Endocrinology and Metabolism 201196 2796-2804. (https://doi. org/10.1210/jc.2011-0536)

Received 7 February 2021

Accepted 2 March 2021 